\title{
The Development of a Novel Technique for Small Ring Specimen Tensile Testing
}

\author{
J. Kazakeviciute, J.P. Rouse, D.S.A. De Focatiis and C.J. Hyde*
}

Faculty of Engineering, University of Nottingham, UK, NG7 2RD;

* Correspondence: Christopher.Hyde@ nottingham.ac.uk; Tel.: +44-115-951-3735

\begin{abstract}
The wide scale use of small specimens in routine testing programs could significantly reduce material resource requirements (factors of 10 are easily achievable). This is a major benefit to situations where there is not enough material to manufacture conventional, full-size specimens, such as first-stage gas turbine blade roots. However, limitations exist due to concerns over size effects, manufacturing difficulties, uncertainties related to the application of representative loading conditions and complex interpretation procedures of non-standard data. Due to these limitations, small specimen testing techniques have been mostly applied in ranking exercises and to determine approximate or simple material parameters such as Young's modulus, minimum creep strain rate and fracture toughness. The small ring method is a novel, high sensitivity small specimen technique for creep testing that has been extended in the present work to the determination of tensile material properties. The main advantages of the small ring specimen are that it is self-aligning and has a large equivalent gauge length in comparison to other small specimens, resulting in much higher testing sensitivity. In the present work, this specimen type mimics conventional, full-size, monotonic testing, allowing for observations of elastic and plastic material response to be made. Wrought aluminium alloy 7175-T7153 small rings were tested at room temperature at 5 different loading (displacement) rates and the results compared to conventional, full-size, monotonic specimen equivalents. Finite element analysis was conducted in order to evaluate the equivalent gauge section and equivalent gauge length in the small ring specimen (which varied between circa $0.35-1.4 \mathrm{~mm}^{2}$ and $25-45 \mathrm{~mm}$, respectively) to facilitate these comparisons. An analytical solution has also been derived in order to validate the finite element analysis.
\end{abstract}

Keywords: small ring specimen; tensile testing; finite element analysis; material properties; aluminium.

\section{Introduction}

Small specimens are used in situations where not enough material is available for testing of standard, fullsize specimens, such as when developing a new material, when effects of irradiation are being investigated (test reactors have a limited effective irradiation volume and handling large amounts of irradiated material is undesirable), when investigating the condition of the material of service-aged turbine blade roots [1] or when evaluating additively manufactured materials [2]. Small specimens can be used to acquire properties related to a range of physical phenomenon such as tensile, fatigue [3], fracture [4], and crack propagation [5], with a substantial body of work focused on obtaining creep properties [6, 7].

In general, there are two main types of small specimens used for tensile testing - small punch and miniaturized standard specimens. Small punch specimens have been used for identifying yield strength and ultimate tensile strength [8], while miniaturized standard specimens are suitable for identifying the whole stress-strain curve including Young's modulus [3]. Small punch specimens are typically either square $10 \times 10 \times 0.5 \mathrm{~mm}$ [8] or circular with a diameter between $3 \mathrm{~mm}$ [9] and 10 $\mathrm{mm}$ [10] and a similar thickness to the square specimens. The force-displacement curves of small punch specimens are complicated [11], with many different regions representing different stages of deformation and a complex interplay between, for example, developing friction, contact area, and material response. This makes the data difficult to interpret as the effects of material deformation (the information sought by the experimenter) are masked by other factors. Miniaturized standard specimens vary in size from $0.5 \times 0.3 \times 0.3 \mathrm{~mm}$ gauge section [12] upwards. Despite handling and alignment difficulties arising from the small dimensions, data obtained from miniaturized standard specimens is easier to interpret than that of small punch testing. Manufacture of miniaturised specimens, however, is significantly costlier, usually requiring the welding of end grips on thin gauge sections of test material which introduces an opportunity for unintentional heat treatment of the gauge section material.

The small ring specimen, developed in 2009 by Hyde and Sun [13], allows creep strain data to be obtained and has several advantages over alternative methods, such as having a large equivalent gauge length (providing 
high test sensitivity), being self-aligning and simple to manufacture. The main shortcoming of this specimen type is that it cannot be used to obtain tertiary creep data due to low sensitivity in that region as strain in the small ring specimen is high when tertiary region would be reached (this is shortcoming shared with many of the other small specimen types).

All small ring specimen techniques up to date were focussed on determining creep properties. In this work the applicability of the small ring method has been extended to determining rate independent elastic-plastic behaviour. It was done by identifying a method to extract stress-strain data equivalent to conventional monotonic testing from small ring test force/displacement results. Wrought aluminium alloy 7175-T7153 was used as the demonstrative material in this study where small rings were tested at room temperature at 5 different loading (displacement) rates for which the equivalent gauge section and equivalent gauge length in the small ring specimen were determined by finite element (FE) analysis. A strain energy based analytical solution has also been derived and used to validate the FE solutions and both solutions, along with the small ring experimental data have been compared to experimental data obtained from conventional, full-size, monotonic specimen equivalents.

\section{Nomenclature}

$A$

$A_{\text {gauge }}$

$d x$

$E$

G

I

$L$

$L^{\prime}$

$L_{\text {gauge }}$

$M$

$M_{0}$

$M_{\theta}$

$n$

$P$

$Q$

$R$

$r_{\mathrm{s}}$

$S$

$T$

$U_{\mathrm{b}}$

$u_{\mathrm{h}}$

$U_{s}$

$U_{\mathrm{t}}$

$u_{\mathrm{v}}$

$\alpha$

$\Delta L$

$\varepsilon$

$\varepsilon_{\text {bending }}$

$\varepsilon_{\text {uniaxial }}$

$\theta$

$v$

$\sigma$

$\sigma_{\mathrm{y}}$
Cross-sectional area $\left(\mathrm{m}^{2}\right)$

Equivalent gauge section $\left(\mathrm{m}^{2}\right)$

Horizontal distance between grips (m)

Young's modulus $(\mathrm{Pa})$

Shear modulus $(\mathrm{Pa})$

Second moment of area $\left(\mathrm{m}^{4}\right)$

Length of a standard creep specimen between grips (m)

Distance between pins $(\mathrm{m})$

Equivalent gauge length $(\mathrm{m})$

Bending moment $(\mathrm{Nm})$

Reaction moment $(\mathrm{Nm})$

Moment at angle $\theta(\mathrm{Nm})$

Material constant for Ramberg-Osgood material model

Applied load (N)

Dummy load (N)

Radius of the neutral axis of the small ring specimen (m)

Radius of the cross-section of standard creep specimen (m)

Shear force $(\mathrm{N})$

Tensile force $(\mathrm{N})$

Bending strain energy $(\mathrm{Nm})$

Horizontal displacement (m)

Shear strain energy $(\mathrm{Nm})$

Tensile strain energy $(\mathrm{Nm})$

Vertical displacement (m)

Material constant for Ramberg-Osgood material model

Change in length (m)

Strain

Bending strain

Uniaxial strain

Angle from applied load P (rad)

Poisson's ratio

Stress $(\mathrm{Pa})$

Yield strength $(\mathrm{Pa})$ 


\subsection{Material}

The material tested was aluminium alloy 7175-T7153, with the composition shown in Table 1. This material was chosen as it is being considered for aircraft gearbox housing applications. This material is a heat treated wrought alloy and specimens were machined from a hot-rolled plate.

Table 1. Composition of aluminium alloy 7175-T7153 [14].

\begin{tabular}{ccccccccccc}
\hline Name & $\mathbf{Z n}$ & $\mathbf{M g}$ & $\mathbf{C u}$ & $\mathbf{C r}$ & $\mathbf{T i}$ & $\mathbf{F e}$ & $\mathbf{M n}$ & $\mathbf{S i}$ & Other & Al \\
\hline $\mathrm{wt} \%$ & 5.7 & 2.5 & 1.6 & 0.2 & 0.04 & 0.06 & 0.02 & 0.03 & 0.02 & $\mathrm{Bal}$ \\
\hline
\end{tabular}

The grain size of the material is $\sim 250 \mathrm{~nm}^{2}$, found from TEM images of the material, an example of which can be seen in Figure 1. Precipitates are zinc and magnesium-rich particles dispersed through the matrix, copperrich particles are on the grain boundaries and chromium-rich particles are within the grains near the boundary. The average particle size is $\sim 200 \mathrm{~nm}^{2}$ [14]. The grain size is small enough that this material can be used for small ring specimen testing, as there is a sufficient number of grains through each dimension of the crosssection.

\subsection{Experimentation}

Monotonic testing of conventional, full-size specimens, at room temperature, for a range of loading (displacement) rates was carried out in order to investigate whether any rate dependency exists within the material. The loading rates used for this testing were $10 \mathrm{~mm} / \mathrm{min}, 1 \mathrm{~mm} / \mathrm{min}$ [14] and $0.1 \mathrm{~mm} / \mathrm{min}$. The $1 \mathrm{~mm} / \mathrm{min}$ loading rate tests were carried out on an Instron 5985 testing machine fitted with a 100kN load cell [14], whilst all other loading rate tests were carried out on a Mayes 250 testing machine fitted with a $25 \mathrm{kN}$ load cell.

The small ring specimen testing was carried out at loading (displacement) rates of $60.0 \mathrm{~mm} / \mathrm{min}$, $6.00 \mathrm{~mm} / \mathrm{min}, 2.04 \mathrm{~mm} / \mathrm{min}, 0.204 \mathrm{~mm} / \mathrm{min}$ and $0.0204 \mathrm{~mm} / \mathrm{min}$. The first two rates were chosen according to the work of Hyde and Sun [13], while the rest were chosen according to preliminary FE analyses. The three lowest rates were tested on the Mayes testing machine mentioned above, whilst all other rates were performed on a Tinius Olsen H25KS testing machine fitted with a $25 \mathrm{kN}$ load cell. In order to evaluate vertical displacement LVDT extensometer readings from knife edges were used. In order to evaluate horizontal displacement of the ring, video data from a FLIR SC7200 infrared camera was used, collected from the $600.0 \mathrm{~mm} / \mathrm{min}$ and $60.00 \mathrm{~mm} / \mathrm{min}$ tests, both performed on Tinius Olsen H25KS testing machine. In order to further validate the FEA model an interrupted test was done, during which the small ring specimen was deformed by $1.6 \mathrm{~mm}$ and then unloaded. The specimen before testing and after interrupted test and after failure can be seen in Figure 2.

In agreement with Hyde's work [13], the small ring specimen has internal and external diameters of $9 \mathrm{~mm}$ and $11 \mathrm{~mm}$ respectively, a thickness of $2 \mathrm{~mm}$, and is loaded at diametrically opposite points on the vertical axis at the inner circumference by two Nimonic pins, $2.5 \mathrm{~mm}$ in diameter (as shown in Figure 3 ). It is loaded using a modified standard creep specimen.

\subsection{Finite Element model}

Taking advantage of three planes of symmetry within the small ring test setup, a finite element model of $1 / 8^{\text {th }}$ of the ring was developed with appropriate boundary conditions applied on each plane of symmetry, shown in Figure 4(a). The loading pin was modelled as a discrete rigid body because of the significantly larger stiffness of the pin relative to the ring material tested (Nimonic compared to Aluminium). ABAQUS/Standard was used for this modelling, with geometric nonlinearity during the loading step to account for the large deformations and a significantly developing stiffness matrix.

A vertical displacement of $2 \mathrm{~mm}$ (equivalent to a displacement of $4 \mathrm{~mm}$ during experimental testing) was applied to the reference point on the rigid pin, as shown in Figure 4(a) in order to deform the ring. This displacement was chosen as it is approximately the deformation at which all small rings experimentally tested under tensile loading have failed. The selected constitutive model is rate independent since the material tested does not exhibit rate dependent behaviour as shown later in Section 3.

Contact is defined between the pin and the ring, with the pin surface acting as a rigid master surface, and the surface of the ring acting as a slave. ABAQUS surface-to-surface contact was used with Coulomb friction. As experimental measurements of the coefficient of friction were not carried out, a coefficient of friction 
sensitivity study was performed and it was determined that for the deformations of interest (up to $2 \mathrm{~mm}$ in the FEA), the coefficient of friction had almost no effect on stress, strain or reaction force on the pin when varied between 0 and 1 . The model was therefore initialised with a frictionless contact.

In all cases, ABAQUS C3D20R elements were used for meshing the small ring. An investigation was carried out into whether plane stress elements could be used and it was determined that there is a significant variation in stress through the thickness of the ring (likely caused by contact between the ring and the pin), making this element type unsuitable. Quadratic elements were therefore chosen due to the large deformation experienced by the ring and the fact that it is curved, making a quadratic function more suitable to describe the shape than a linear equivalent. Reduced integration was chosen in order to reduce computation time and avoid shear locking. A mesh sensitivity study was undertaken to determine adequate convergence (less than $2 \%$ difference from the response of previous increment of mesh density) with respect to the force-displacement response at an acceptable computation time. The convergence of a variety of outputs was investigated, including stress at a specific displacement, strain at a specific displacement and force on the pin, which can be seen in Figure 5. Mesh density was kept uniform in the small ring specimen, where an element size of $0.2 \mathrm{~mm}$ was used. As for the pin, the element size is larger, but it does not have a significant effect on the model outputs of interest. The elements used to mesh the pin were R3D4. Both meshes can be seen in Figure 4(b).

In order to be able to compare stress-strain data from small ring tensile tests with that of conventional, full-size, monotonic tests an equivalent gauge length and gauge section must be determined. The process was as follows: stress and strain in the vertical direction were extracted from the corner of the ring model (highlighted in Figure 4(b)). The maximum principal stress in this location aligned with the vertical direction, which can be seen in Figure 6, and other principal stresses were small in comparison, suggesting a uniaxial stress state. It is also the location of onset of plasticity due to bending and tensile stress distributions, Reaction force and displacement in the y direction were extracted from the reference point of the pin.

The equivalent gauge section $A_{\text {gauge }}$ and the equivalent gauge length $L_{\text {gauge }}$ were defined using equations (1) and (2), respectively [13]:

$$
\begin{aligned}
& L_{\text {gauge }}=\frac{\Delta L}{\varepsilon}, \\
& A_{\text {gauge }}=\frac{P}{\sigma},
\end{aligned}
$$

where $\Delta L$ is the increment in length acquired from the reference point of the loading pin, $\varepsilon$ is strain in the vertical direction (taken from the FEA model), $P$ is the applied load acquired from the reference point of the pin and $\sigma$ is stress in the vertical direction (taken from the FEA model).

The shapes of the force-displacement curves with an elastic, and an elastic-plastic hardening model, are shown in Figure 7. The figure shows the effect of material non-linearity, therefore the initial part where the plots match can be considered elastic, and beyond where they diverge the plot of the elastic-plastic response can be considered plastic behaviour.

In order to be able to use the uniaxial tensile data in ABAQUS FEA, the Ramberg-Osgood material model (deformation plasticity in ABAQUS) was fitted to it in the form of

$$
\varepsilon=\frac{\sigma}{E}+\alpha \frac{\sigma}{E}\left(\frac{\sigma}{\sigma_{\mathrm{y}}}\right)^{n-1}
$$

where $E$ is Young's modulus, $\sigma_{\mathrm{y}}$ is yield stress and $\alpha$ and $n$ are Ramberg-Osgood material model constants. At strains larger than the ones defined by the material model, material behaviour is assumed to be perfectly plastic. Model parameters were fitted to uniaxial data using the Matlab optimization toolbox and are shown in Table 2. Poisson's ratio was determined experimentally using digital image correlation from a single test and therefore a sensitivity study was carried out in order to investigate the effect of this parameter on the model outputs of interest. It was discovered that there was no significant effect, and hence a Poisson's ratio value of 0.3 was used, as was measured experimentally [14]. Material of the ring was assumed to be isotropic, as shown previously [14]. As the pins are defined as discrete rigid bodies, their material properties did not need to be defined.

Table 2. Material parameters from Ramberg-Osgood material model.

\begin{tabular}{cccccc}
\hline Material & $\begin{array}{c}\text { Temperature } \\
\left({ }^{\circ} \mathbf{C}\right)\end{array}$ & $\begin{array}{c}\mathbf{E} \\
(\mathbf{G P a})\end{array}$ & $\begin{array}{c}\sigma_{\mathbf{y}} \\
(\mathbf{M P a})\end{array}$ & $\alpha$ & $n$ \\
\hline
\end{tabular}


In order to investigate the effect of misalignment a full ring model was modelled. The model setup, shown in Figure 8, was broadly the same as in the case of the $1 / 8^{\text {th }}$ of the ring model, except that the maximum displacement was $4 \mathrm{~mm}$, coefficient of friction was set to 0.3 (the effect of this negligible), automatic stabilisation was on and surface smoothing was on. Mesh size was also the same as for the $1 / 8^{\text {th }}$ model. Misalignments of 5 degrees in the pitch and yaw directions of the top pin relative to the original direction were investigated.

The third type of misalignment, when pins are not in line, as per Figure 9(a) was investigated analytically. As this tensile testing technique is being developed with a view to extend it to fatigue testing, the load train is aligned to Class 5 alignment of BS ISO 23788:2012. It specifies that the maximum misalignment for an alignment cell is that strain due to bending is either 50 microstrain at zero force or $5 \%$ of applied uniaxial strain, whichever one is bigger, which is expressed in an equation as

$$
\begin{gathered}
\varepsilon_{\text {uniaxial }} \times 5 \% \geq \varepsilon_{\text {bending }} . \\
\varepsilon_{\text {uniaxial }}=\frac{\sigma}{E}=\frac{P}{A E}=\frac{P}{\frac{\pi r_{\mathrm{s}}^{2}}{4} E}=\frac{4 P}{\pi r_{\mathrm{s}}^{2} E} \\
\varepsilon_{\text {bending }}=\frac{M r_{S}}{I E}=\frac{P L \sin \theta r_{\mathrm{s}}}{\frac{\pi r_{\mathrm{S}}^{4}}{4} E}=\frac{4 P L \sin \theta}{\pi r_{\mathrm{s}}^{3} E} .
\end{gathered}
$$

After substituting the expressions for uniaxial and bending strain according to Figure 9(b) the result is

$$
\frac{4 P}{\pi r_{\mathrm{s}}^{2} E} \times 5 \% \geq \frac{4 P L \sin \theta}{\pi r_{\mathrm{s}}^{3} E}
$$

which is then simplified to

$$
5 \% \geq \frac{L \sin \theta}{r_{\mathrm{s}}} .
$$

After substituting $L$ as $80 \mathrm{~mm}$, from the length between the grips for a standard creep specimen, used as an alignment cell in this case, and $r_{s}$ as $5 \mathrm{~mm}$, from the radius of the gauge section of the standard creep specimen the misalignment due to pins not being in line is,

$$
\theta \leq 0.179^{\circ}
$$

As shown in Figure 9(b) this results in the maximum $d x$ value of

$$
d x=L \sin \theta=0.05 \mathrm{~mm}
$$

This then needs to be converted to $\theta^{\prime}$ as follows

$$
\begin{gathered}
L^{\prime}=\left(2 R-2 r_{\text {pin }}\right) \times \cos \theta^{\prime} \\
\sin \theta^{\prime} \cos \theta^{\prime}=\frac{1}{2} \sin \left(2 \theta^{\prime}\right)=\frac{d x}{2 R-2 r_{\text {pin }}} . \\
\theta^{\prime}=\frac{1}{2} \arcsin \left(\frac{d x}{R-r_{\text {pin }}}\right) .
\end{gathered}
$$

The resulting maximum $\theta^{\prime}$ is 0.8815 degrees, which results in less than $1 \%$ error in force and displacement measurements.

\subsection{Analytical solution}

An analytical elastic solution based on a strain energy solution method was developed. It was assumed that the ring can be considered as a quarter circular slender beam (the stress through the thickness of the ring is constant), with the appropriate boundary conditions applied as:

- No displacement or rotation of point A,

- No rotation of point B. 
The simplified loading and bending moment diagram can be seen in parts a and $\mathrm{b}$ of Figure 10, respectively. In reality, the horizontal displacement measured in the point B in this analytical solution would be present at point $\mathrm{A}$ in the experiment.

Equilibrium gives:

$$
M_{\theta}=M_{0}+P R \sin \theta-Q R(1-\cos \theta),
$$

then, according to the strain energy approach, since complimentary strain energy and strain energy are equal for linear elastic materials,

$$
U^{*}=U_{\mathrm{b}}=\int_{0}^{L} \frac{M_{\theta}^{2}}{2 E I} d x=\int_{0}^{\frac{\pi}{2}} \frac{M_{\theta}^{2}}{2 E I} R d \theta
$$

which is then integrated and results in

$$
U_{\mathrm{b}}=\frac{R}{2 E I}\left(M_{0}^{2} \times \frac{\pi}{2}+P^{2} R^{2} \times \frac{\pi}{4}+Q^{2} R^{2} \times\left(\frac{3 \pi}{4}-2\right)+2 M_{0} P R-P Q R^{2}-M_{0} Q R \times(\pi-2)\right) .
$$

As symmetry of the ring dictates that there should be no rotation at the load $\mathrm{P}$, application point, then

$$
\frac{\partial U_{\mathrm{b}}}{\partial M_{0}}=0=\frac{R}{2 E I}\left(2 M_{0} \frac{\pi}{2}+2 P R-Q R(\pi-2)\right),
$$

therefore,

$$
M_{0}=Q R\left(1-\frac{2}{\pi}\right)-\frac{2}{\pi} P R
$$

Deformation is dominated by bending, but there are also tensile and shear contributions, so after balancing the force in the $\mathrm{x}$ and y directions and solving for $T$ and $S$,

$$
\begin{aligned}
& T=P \sin \theta+Q \cos \theta, \\
& S=P \cos \theta-Q \sin \theta .
\end{aligned}
$$

The tensile complementary strain energy is given by,

$$
U_{\mathrm{t}}=\int_{0}^{L} \frac{T^{2}}{2 A E} d x=\int_{0}^{\frac{\pi}{2}} \frac{T^{2}}{2 A E} R d \theta,
$$

which is then integrated and results in

$$
U_{\mathrm{t}}=\frac{R}{2 A E}\left(\frac{\pi}{4} Q^{2}+P Q+\frac{\pi}{4} P^{2}\right) .
$$

Shear complementary strain energy is given by

$$
U_{\mathrm{s}}=\int_{0}^{L} \frac{s^{2}}{2 A G} d x=\int_{0}^{\frac{\pi}{2}} \frac{s^{2}(1+v)}{A E} R d \theta,
$$

which is then integrated and results in

$$
U_{\mathrm{S}}=\frac{R(1+v)}{A E}\left(\frac{\pi}{4} P^{2}-P Q+\frac{\pi}{4} Q^{2}\right) .
$$

Equation (4) is subbed back into Equation (3) to get an expression that can be differentiated by $P$ and $Q$. As the vertical displacement is obtained by differentiating total strain energy by $P$, it can be expressed as,

$$
u_{\mathrm{v}}=\frac{\partial U_{\mathrm{b}}}{\partial P}+\frac{\partial U_{\mathrm{t}}}{\partial P}+\frac{\partial U_{\mathrm{s}}}{\partial P}=\frac{P R^{3}}{E I}\left(\frac{\pi}{4}-\frac{2}{\pi}\right)+\frac{P R \pi}{4 A E}+\frac{P R \pi}{2 A E}(1+v)
$$

while the horizontal displacement is obtained by differentiating by the dummy load $Q$,

$$
u_{\mathrm{h}}=\frac{\partial U_{\mathrm{b}}}{\partial Q}+\frac{\partial U_{\mathrm{t}}}{\partial Q}+\frac{\partial U_{\mathrm{s}}}{\partial Q}=\frac{P R^{3}}{2 E I}\left(1-\frac{4}{\pi}\right)+\frac{P R}{2 A E}-\frac{P R}{A E}(1+v) .
$$

\section{Results}

Figure 11(a) shows the uniaxial stress-strain experimental results, with no significant rate dependency demonstrated. The results were truncated at around $6 \%$ strain due to uniaxial specimens failing over a large range of strains starting at around 6\%. The Ramberg-Osgood model is also shown and it can be seen that the model fits well in both the elastic and plastic regions, with a slight deviation around the yield point. Figure 
11(b) shows the force-vertical displacement results from small ring tensile testing and as in uniaxial tensile testing, there is no noticeable rate dependency at these loading rates. These force-displacement curves have interesting and sharp features in them at the later stages due to cracks developing in the specimens and therefore deviating from a material (continuum) test (best seen in $60 \mathrm{~mm} / \mathrm{min}$ rep2). Figure 11(c) shows the same trends for force-horizontal displacement results.

Figure 12(a) shows a comparison of the small ring force-vertical displacement data obtained experimentally with both the FEA simulation and the elastic analytical solution shown in Equation (5). It can be seen that the agreement between the three data sets is extremely close (with the elastic analytical solution deviating at yield point, as would be expected). Figure 12(b) also follows the same pattern of analytical solution shown in Equation (6) matching FEA and experimental data for force-horizontal displacement curve.

Figure 13(a) shows the equivalent gauge section, plotted against reaction force on the loading pin which was used to calculate the stress from the small ring tensile test results in order to compare it to uniaxial stress data, by using Equation 2. Figure 13(b) shows the equivalent gauge length, plotted against the displacement of the loading pin, which can then be used to calculate strain from the small ring tensile test in order to compare it to uniaxial strain data, by using Equation 1. Figure 14 shows the comparison between the shape of the small ring specimen after interrupted test and a small ring specimen FEA model after being loaded and unloaded in the same way as the interrupted test to further validate the FEA model.

Figure 15 shows an example of the stress-strain data reconstructed from the small ring experimental data and the equivalent gauge section and length information obtained, compared with the measured stress-strain data from standard, full-size monotonic testing. The agreement is extremely good, with a small deviation around the yield point. Similarly to uniaxial stress/strain results, these calculated results have also been truncated due to small ring specimens failing over a large range of displacements and the fact that high strain behavior is not clear due to the effect of pins.

\section{Discussion}

As was shown in Figure 11(a), uniaxial stress-strain measurements were found to be repeatable and rate independent, which suggests that the material is uniform and that the testing machine and methodology are suitable for this test. The lack of strain rate dependence also in small ring tensile tests shown in Figure 11(b) is consistent with the uniaxial behavior of this aluminium alloy at room temperature. A Ramberg-Osgood material model was chosen because it is commonly used in FEA. It fits reasonably well, having the $\mathrm{R}^{2}$ values of between 0.8655 and 0.9907 for the 7 datasets presented, with a slight deviation in fitting quality around the yield point, possibly because the maximum strain to which it is fitted is too large. This large strain was selected for the material model because the small ring experiences significant strains during tensile testing, and therefore the ability to represent large strains is important.

Small ring tensile test results were also shown to be repeatable, as shown in Figure 11(b), and rateindependent, as with the uniaxial testing equivalents. This suggests that it ought to be possible to compare results from small ring tensile testing with uniaxial testing with the proper geometric corrections. The irregular steps observed in some of the force-displacement curves arise because of partial cracking, which becomes progressively more severe at larger displacements, until the rings eventually fail (i.e. become non-elliptical or fracture).

The small ring FE solution was validated by the elastic analytical solution in the elastic region of the forcedisplacement curve. The FE solution also matches experimental data well, even at larger displacements, and is found to be suitable for modelling this test.

The equivalent gauge section determined from equation (1) and shown in Figure 13(a) is constant in the elastic region, up to about $140 \mathrm{~N}$ of applied load for the material tested, and steadily increases in the plastic region. The equivalent gauge length determined from equation (2) and shown in Figure 13(b) starts from $\sim 45$ $\mathrm{mm}$, falls to $\sim 40 \mathrm{~mm}$ by the end of the elastic region which is at $\sim 0.25 \mathrm{~mm}$ of vertical displacement and progressively falls through to a minimum of $\sim 25 \mathrm{~mm}$. When employing the equivalent gauge section and length to compute stress-strain data from the small ring tests, a good match was found between the computed small ring and the measured uniaxial test results, as shown in Figure 15, with a slight deviation around yield, likely caused by the limited fit of the Ramberg-Osgood material model around the yield stress. It is possible that an inverse analysis could be used to obtain even more accurate stress-strain data, as suggested in [16]. 
The effect of pin misalignment was also investigated using FEA, and is shown in Figure 16 for both the pitch and yaw directions. A value of 5 degrees was selected as the magnitude to be investigated because it is enough to be visible with the naked eye in the experimental setup, and can therefore be rectified before testing. Neither yaw nor pitch misalignment (of this magnitude) were found to have a significant effect on the results. It is concluded that pin misalignment does not have a significant effect on the results provided it can be kept to within small values. The potential of a small amount of misalignment in the small ring experimental data presented is therefore considered insignificant.

\section{Conclusions}

- $\quad$ This paper describes the use of a small ring test to produce equivalent stress-strain data for a wrought aluminium alloy at room temperature. An elastic analytical solution was obtained from a strain energy approach, and a finite element mesh was set up to model the ring displacement using a Ramberg-Osgood material model fitted to uniaxial stress-strain data. The ring displacements obtained using the FE solution were in excellent agreement with those found in experiment, and, in the elastic region, also with those from the analytical solution.

- Functions of the equivalent gauge section and length were obtained using the FE simulations for the purpose of reconstructing the stress-strain data from the small ring tensile experiments.

- The small ring tensile experiments were found to be suitable for performing tensile tests, since the material is isotropic and the ring is symmetrical. The scatter in the small ring tensile testing is small, and similar to that obtained in the tensile test data when the stress-strain curves are computed from the force-displacement curves using the equivalent gauge length and section information.

- Testing and simulation at elevated temperature will likely require the introduction of rate dependence in the model, since, at sufficiently high temperature, this material exhibits noticeable rate dependence [14].

- It is intended that the developed small ring tensile test technique be applied a selection of different materials, including steel and nickel superalloys, in order to explore whether the shape of the stress-strain curve is an important factor in the computation of equivalent gauge dimensions.

Acknowledgments: The authors wish to acknowledge the EPSRC CDT in Innovative Metal Processing (Grant No: EP/L016206/1) for financial support of this project.

\section{References}

[1] C. J. Hyde, T. H. Hyde, W. Sun, S. Nardone, E. De. Bruycker, Small Ring Testing of a Creep Resistant Material, Mater. Sci. Eng., A 586 (2013) 358-366. https://doi.org/10.1016/j.msea.2013.07.081.

[2] Z. Xu, C. J. Hyde, C. J. Tuck, A. T. Clare, Creep Behaviour of Inconel 718 Processed by Selective Laser Melting, J. Mater. Process. Technol. 256 (2018) 13-24. https://doi.org/10.1016/j.jmatprotec.2018.01.040.

[3] D. Holländer, D. Kulawinski et al. Investigation of isothermal and thermo-mechanical fatigue behavior of the nickelbase superalloy IN738LC using standardized and advanced test methods. Mater. Sci. Eng., A 67 (2016) 314-324. https://doi.org/10.1016/j.msea.2016.05.114.

[4] W. Han, K. Yabuuchi et al. Application of small specimen test technique to evaluate fracture toughness of reduced activation ferritic/martensitic steel. Fusion $\quad$ Eng. Des. $125 \quad$ (2017) 326-329. https://doi.org/10.1016/j.fusengdes.2017.01.040

[5] C.-S. Shin, S.-W. Lin, Evaluating fatigue crack propagation properties using miniature specimens, Int. J. Fatigue 43 (2012) 105-110. https://doi.org/10.1016/j.jifatigue.2012.02.018.

[6] T.H. Hyde, C.J. Hyde, W. Sun, Theoretical basis and practical aspects of small specimen creep testing. J. Strain Anal. Eng. Des. 48 (2013) 112-125. https://doi.org/10.1177/0309324712463299.

[7] T. H. Hyde, C. J. Hyde, W. Sun, A Basis for Selecting the Most Appropriate Small Specimen Creep Test Type, J. Pressure Vessel Technol. 136(2) (2014). https://doi.org/10.1115/1.4025864.

[8] T.E. García, B. Arroyo et al., Small punch test methodologies for the analysis of the hydrogen embrittlement of structural steels, Theor. Appl. Fract. Mech. 86 (2016) 89-100. https://doi.org/10.1016/j.tafmec.2016.09.005.

[9] K. Kumar, A. Pooleery et al., Evaluation of ultimate tensile strength using Miniature Disk Bend Test, J. Nucl. Mater. 461 (2015) 100-111. https://doi.org/10.1016/j.jnucmat.2015.02.029. 
[10] M. Song, K. Guan et al., Size effect criteria on the small punch test for AISI 316L austenitic stainless steel, Mater. Sci. Eng., A 606 (2014) 346-353. https://doi.org/10.1016/j.msea.2014.03.098.

[11] T. H. Hyde, M. Stoyanov, W. Sun, C. J. Hyde, On the Interpretation of Results from Small Punch Creep Tests, J. Strain Anal. Eng. Des. 45(3) (2010) 141-164. https://doi.org/10.1243/03093247JSA592.

[12] K. Kumar, A. Pooleery et al., Optimisation of thickness of miniature tensile specimens for evaluation of mechanical properties, Mater. Sci. Eng., A 675 (2016) 32-43. https://doi.org/10.1016/j.msea.2016.08.032.

[13] T.H. Hyde, W. Sun, A novel, high-sensitivity, small specimen creep test, J. Strain Anal. Eng. Des. 44 (2009) 171-185. https://doi.org/10.1243/03093247JSA502.

[14] M.F. Lam Wing Cheong, Experimental and Numerical Investigations into the Behaviour of a 7175-T7351 Aluminium Alloy for Aerospace Gearbox Housing Applications at Elevated Temperatures, PhD, University of Nottingham, Nottingham, UK, 2018.

[15] M.F. Lam Wing Cheong, J.P. Rouse, C.J. Hyde, A.R. Kennedy, The Prediction of isothermal cyclic plasticity in 7175 T7351 aluminium alloy with particular emphasis on thermal ageing effects, Int. J. Fatigue 114 (2018) 92 - 108. https://doi.org/10.1016/j.ijfatigue.2018.05.010.

[16] A. Husain, D.K. Sehgal, R.K. Pandey, An inverse finite element procedure for the determination of constitutive tensile behavior of materials using miniature specimen, Comput. Mater. Sci. 31 (2004) 84-92. https://doi.org/10.1016/j.commatsci.2004.01.039. 


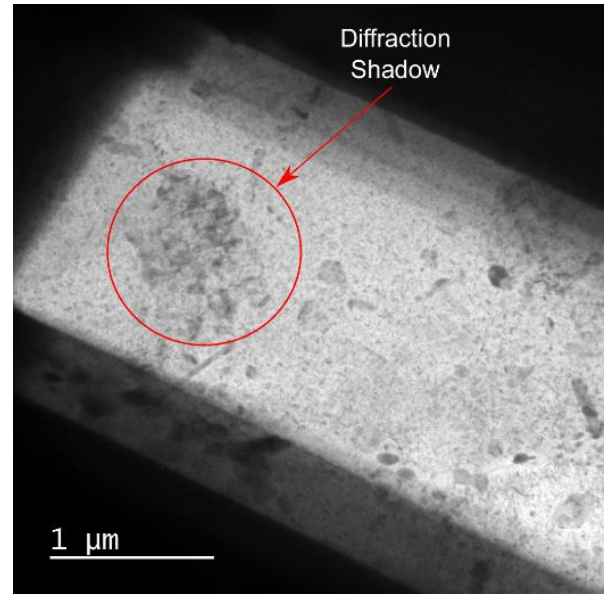

Figure 1. TEM image of the aluminium alloy 7175-T7153 used in this study [15].

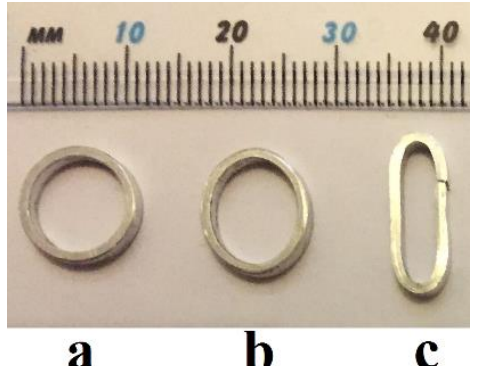

Figure 2. Examples of small specimens (a) Before testing; (b) Interrupted test; (c) After specimen failure.

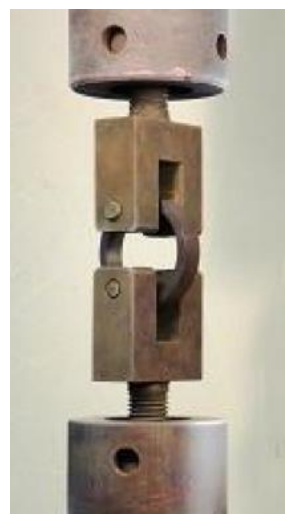

Figure 3. Small ring specimen testing setup.

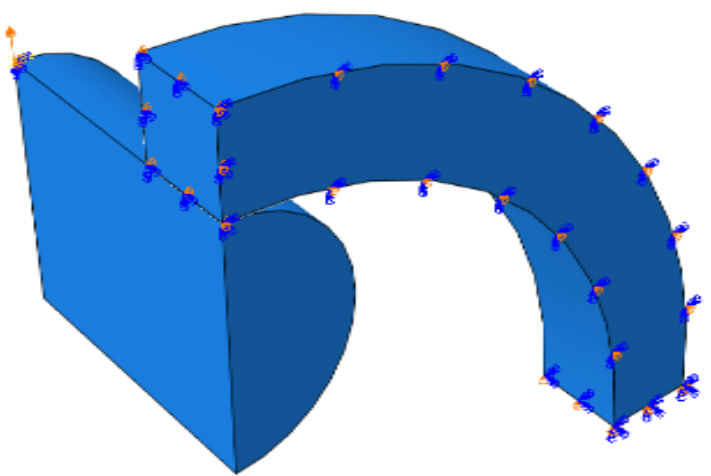

(a)

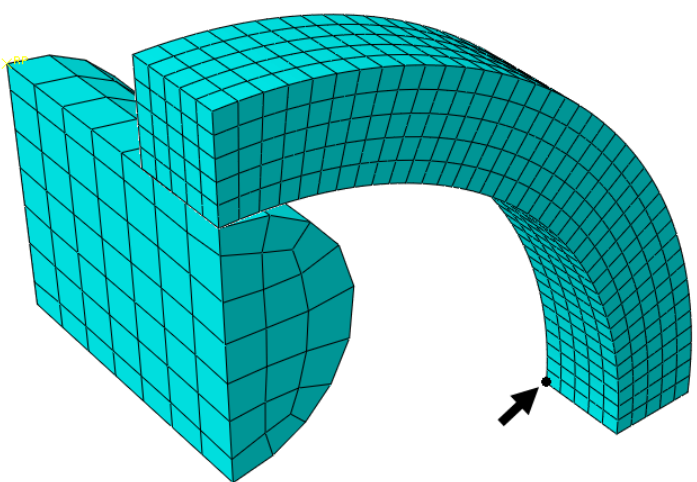

(b)

Figure 4. (a) Loads and boundary conditions in the small ring and loading pin FEA model; (b) Mesh in the small ring and loading pin model. The point from which stress and strain were extracted for equivalent gauge section and length calculations is highlighted by the dot and arrow. 


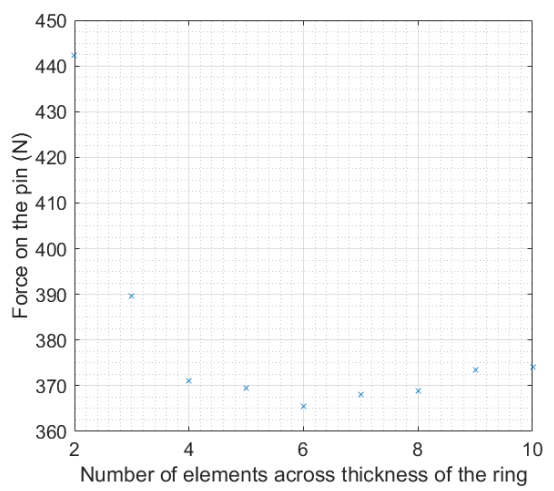

Figure 5. Mesh convergence plot of force on the loading pin.

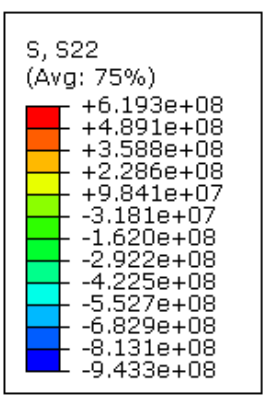

(a)

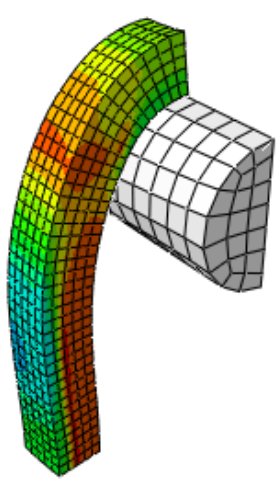

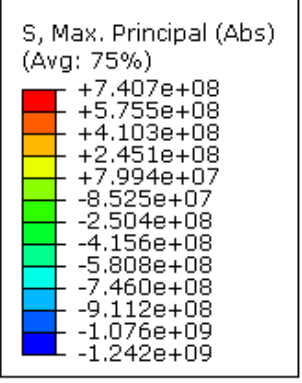

(b)

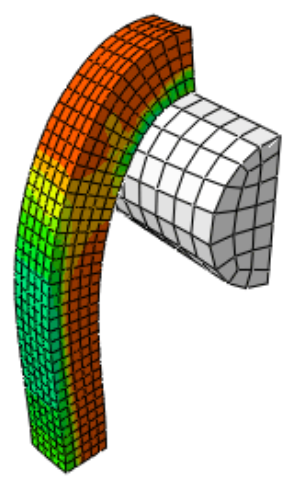

Figure 6. (a) Stress in the y direction in the small ring specimen; (b) Maximum principal stress in the small ring specimen.

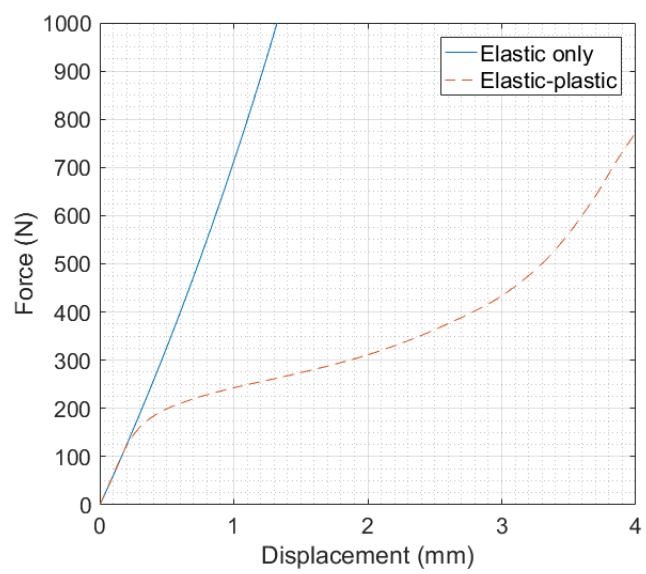

Figure 7. Comparison of the force-displacement at the pin using an elastic material model and an elasticplastic material model. 

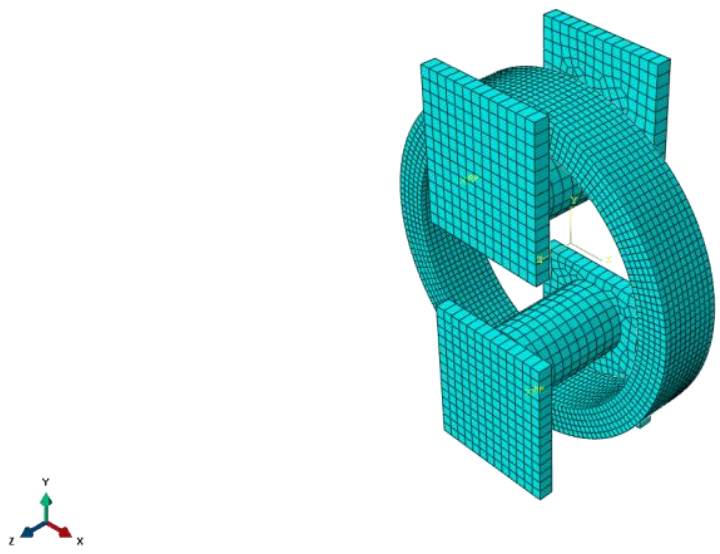

Figure 8. FE mesh of a full ring model.

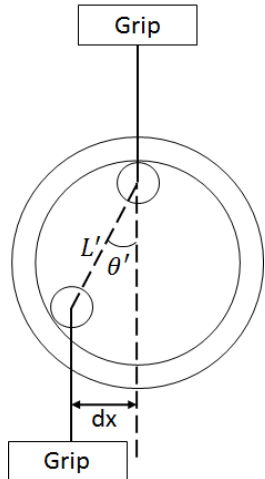

(a)

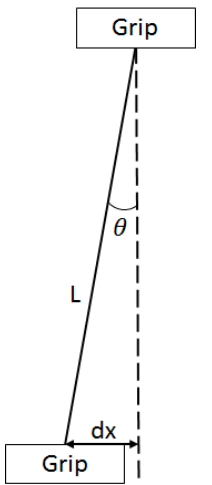

(b)

Figure 9. (a) Misalignment of small ring specimen when pins are not in line; (b) Misalignment of a standard specimen.

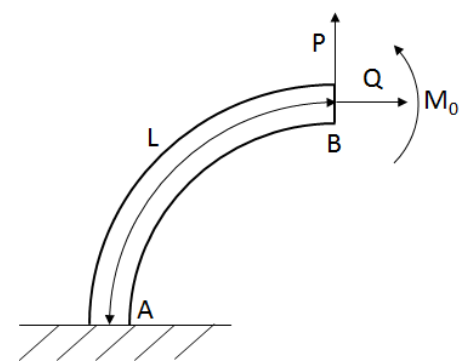

(a)

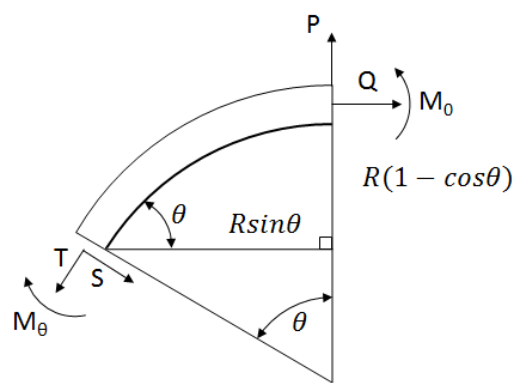

(b)

Figure 10. (a) Simplified loading for analytical solution; (b) Free-body diagram used for strain energy based analytical solution.

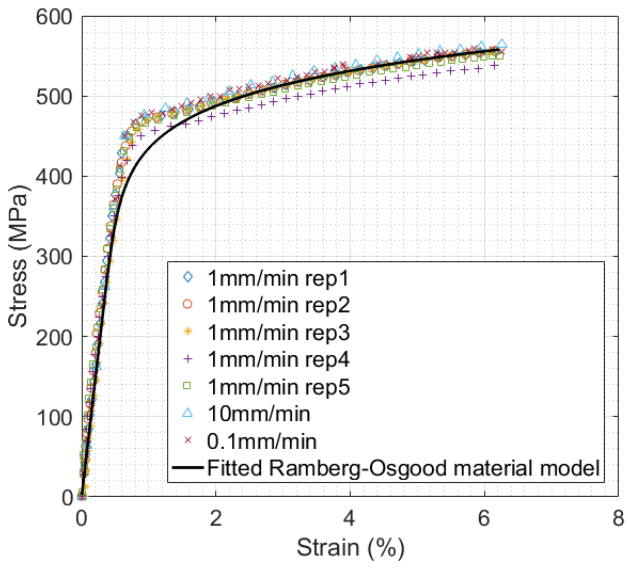

(a)

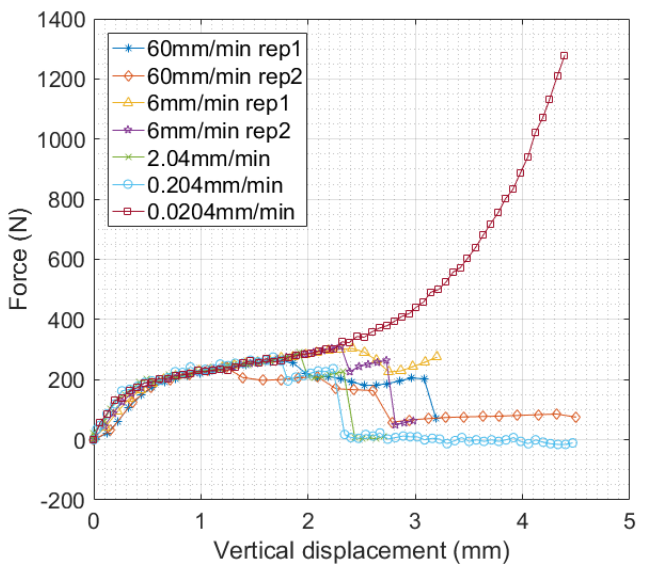

(b) 


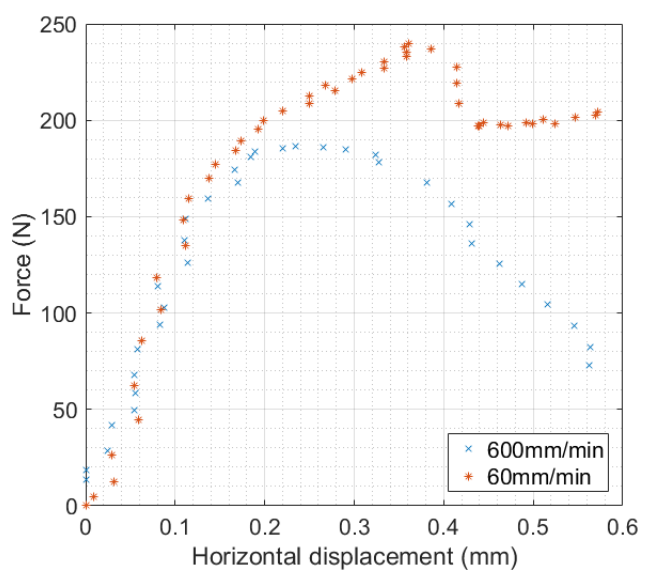

(c)

Figure 11. (a) True stress-true strain curves from the uniaxial experimental tensile testing and fitted Ramberg-Osgood material model; (b) Force-vertical displacement curves from the small ring experimental tensile testing; (c) Force-horizontal displacement curves from small ring experimental testing

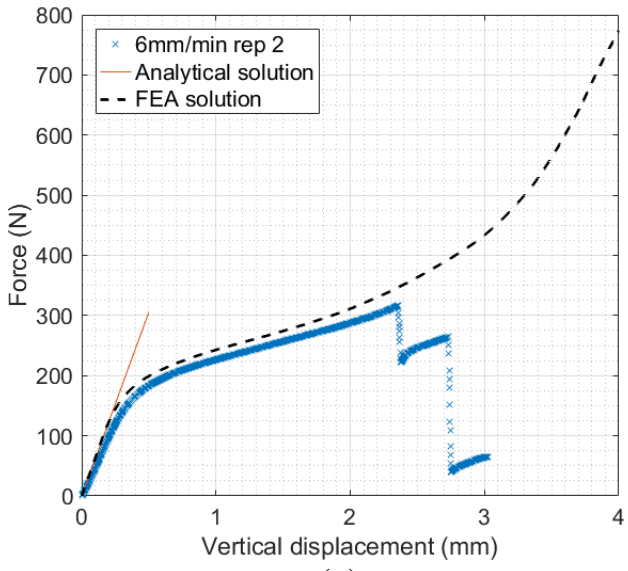

(a)

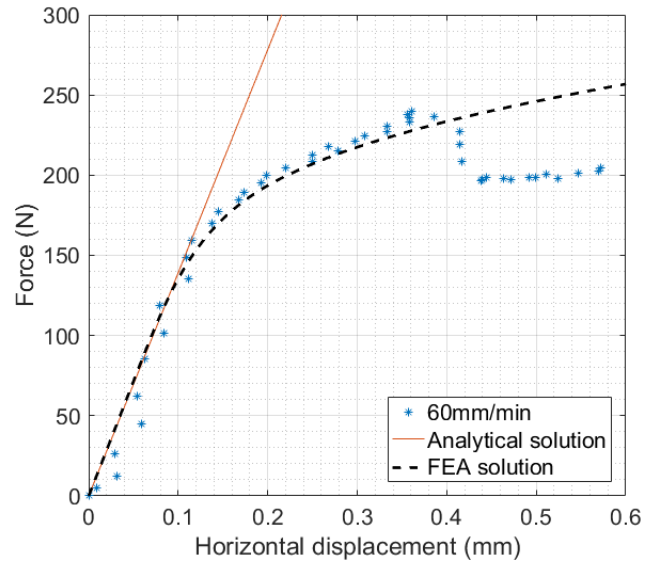

(b)

Figure 12. Small ring force-displacement data obtained from experimentation, FE simulation and the elastic analytical solution for (a) vertical displacement, as described by equation 14 and (b) horizontal displacement, as described by equation 15 .

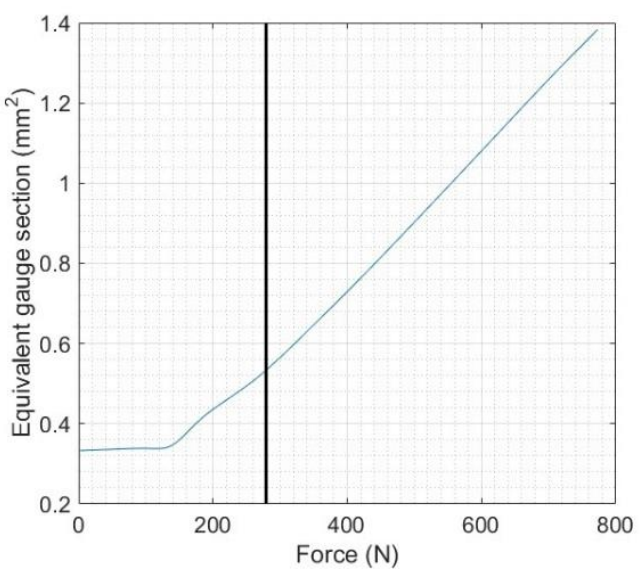

(a)

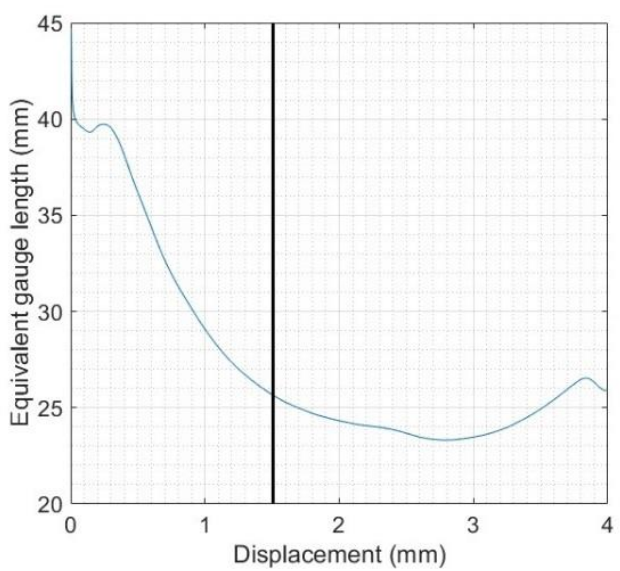

(b)

Figure 13. (a) Equivalent gauge section calculated from FEA plotted against pin force, perfectly plastic after the line; (b) Equivalent gauge length calculated from FEA plotted against pin displacement, perfectly plastic after the line. 

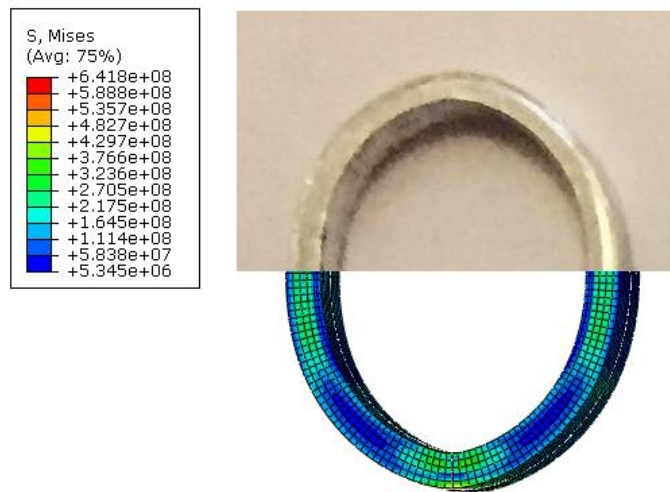

Figure 14. Comparison of shapes of an interrupted test small ring specimen and an unloaded FEA model.

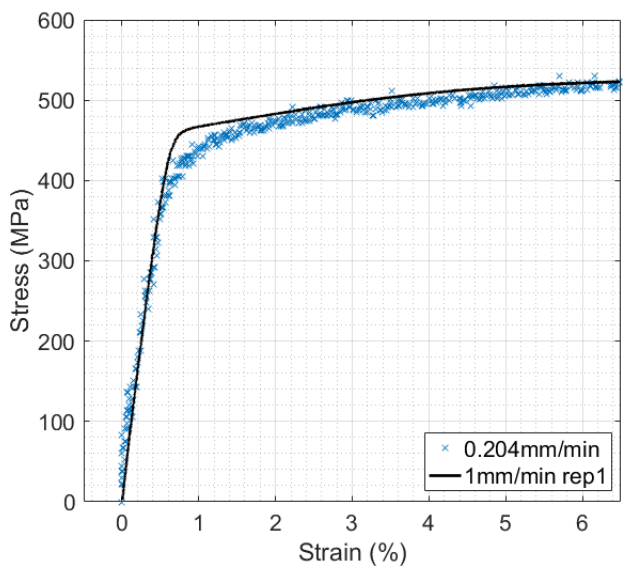

Figure 15. Comparison of stress-strain data from uniaxial testing and stress-strain data calculated from small ring tensile test data and length and equivalent gauge and section.

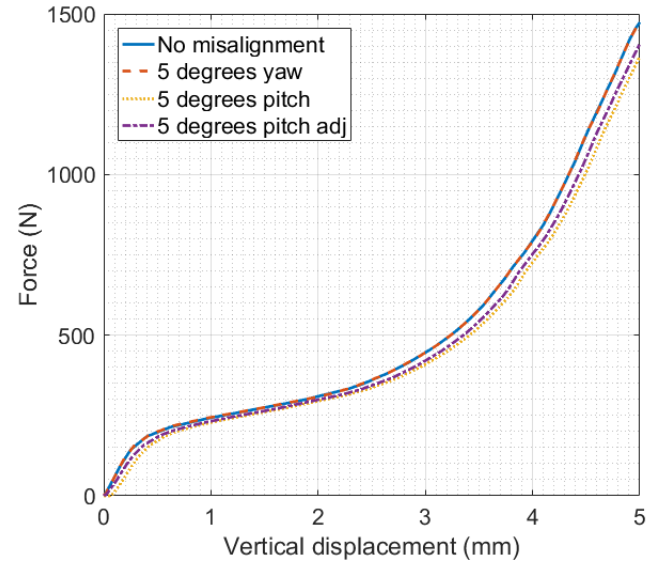

Figure 16. Force-displacement plots showing the effect of 5 degrees yaw and pitch misalignment. 